\title{
Bioinformatics: An Introduction
}

\author{
Nidhi Mishra* \\ Department of Applied Sciences, Indian Institute of Information Technology, India
}

*Corresponding author: Nidhi Mishra, Department of Applied Sciences, Indian Institute of Information Technology-Allahabad, Deoghat, Jhalwa, UP211012, India.

Submission: 眥April 16, 2018; Published: 眥 May 11, 2018

\section{Opinion}

An unprecedented revolution has been observed in science with recent technological advances, which have provided a large amount of "omic" data. The crescent generation and availability of this information available in public databases were, and still are, a challenge for professionals from different areas. However, what is the challenge? In biology, the main challenge is to make sense of the enormous amount of structural data and sequences that have been generated at multiple levels of biological systems. Still, in bioinformatics, development of tools is necessary (statistical and computational) capable of assisting in understanding the mechanisms underlying biological questions in the study. Besides, if we consider the complexity of science, this is a highly reductionist view. The era of a "new biology" emerges accompanied by the birth/development of other sciences, such as bioinformatics and computational biology, which have an integrated interface of molecular biology. Although considered recently, bioinformatics and genomics have evolved interdependently and promoted a historical impact on the available knowledge.

Bioinformatics, a hybrid science that links biological data with techniques for information storage, distribution and analysis to support multiple areas of scientific research including biomedicine. It mainly involving molecular biology and genetics, computer science, mathematics and statistics. Data intensive, large-scale biological problems are addressed from a computational point of view. Bioinformatics is fed by high-throughput data-generating experiments, including genomic sequence determinations and measurements of gene expression patterns. Database projects curate and annotate the data and then distribute it via the World Wide Web. Mining these data leads to scientific discoveries and to the identification of new clinical applications.

A bioinformatics solution usually involves the following steps:

A. Collect statistics from biological data

B. Build a computational model
C. Solve a computational modeling problem

D. Test and evaluate a computational algorithm

It also addresses the following aspects:

a. Types of biological information and databases

b. Sequence analysis and molecular modeling

c. Genomic analysis

d. Systems biology

In the field of medicine in particular, a number of important applications for bioinformatics have been discovered. For example, it is used to identify correlations between gene sequences and diseases, to predict protein structures from amino acid sequences, to aid in the design of novel drugs, and to tailor treatments to individual patients based on their DNA sequences (pharmacogenomics). In bioinformatics, we can now conduct global analyses of all the available data with the aim of uncovering common principles that apply across many systems and highlight novel features. The journal "Open Acc Biostat Bioinform" is a nice platform for representing research work and innovations. It is an open access journal and invites articles from all aspects of bioinformatics and biostatistics. Please contribute articles to this journal for better technological exchange of research work, ideas and methodologies. The journal is available online.

\section{Acknowledgement}

I was given the opportunity to write an editorial for this esteemed journal, I acknowledge and appreciate the contribution of all authors, reviewers, editorial board members and other technical and non technical team members. It is inspired by various reviews etc. I would like to thank Mr. Puneet Kumar for his valuable support in writing this editorial. 
Creative Commons Attribution 4.0

International License

For possible submissions Click Here

Submit Article

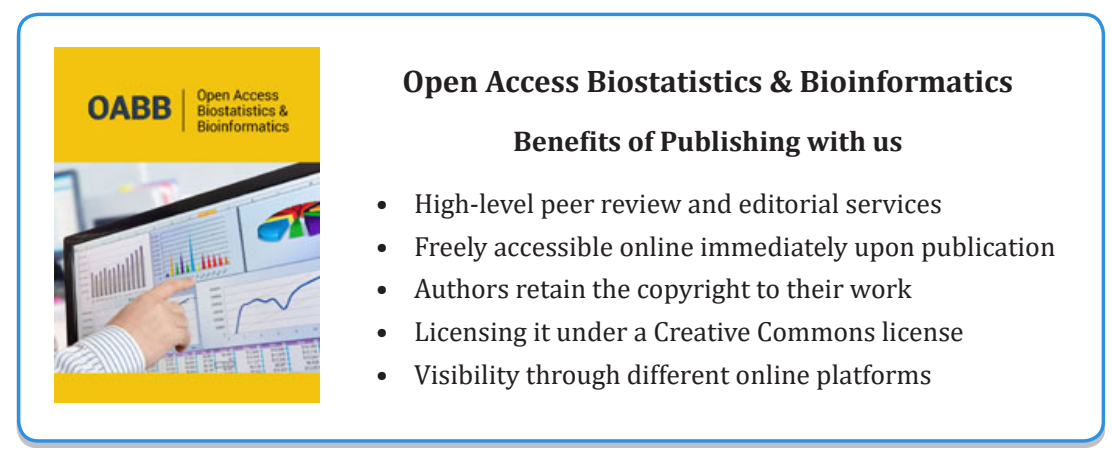

